\title{
Optical phase conjugation in third-order nonlinear photonic crystals
}

\author{
Ping Xie and Zhao-Qing Zhang \\ Department of Physics and Institute of Nano Science and Technology (INST), The Hong Kong University of Science and Technology, \\ Clear Water Bay, Hong Kong, China
}

(Received 13 October 2003; published 10 May 2004)

\begin{abstract}
We predict that the efficiency of the optical phase conjugation generation can be enhanced by more than four orders of magnitude in a $\chi^{(3)}$ nonlinear superlattice, as compared with that in a homogeneous nonlinear medium of the same sample thickness and $\chi^{(3)}$ nonlinearity. Such an effective enhancement utilizes the localized properties of the fields inside the sample at the band-edge state, gap-soliton state, or defect state. Due to the presence of feedback mechanism at each interface of a superlattice, we also predict that the phase conjugation can still be effectively generated when only one pump wave is used.
\end{abstract}

DOI: 10.1103/PhysRevA.69.053806

Photonic crystals have been under intensive studies over the past decade [1]. Since a photonic crystal can possess spectral gaps for frequencies in which light cannot propagate, it makes the control and manipulation of light propagation possible and can have vast implications in both quantum optics and optical devices. To employ the high-technology potential of photonic crystals, it has been recently turned to nonlinear photonic crystals [2]. In a $\chi^{(2)}$ nonlinear photonic crystal the efficiency of the second-harmonic or sumfrequency generation can be enhanced significantly by use of the simultaneous availability of field localization and phasematching condition near the band edge [3]. In a $\chi^{(3)}$ nonlinear photonic crystal, previous studies have been focused on the effects related to the refractive-index change caused by the light intensity via Kerr effect. For example, by changing the light intensity at a frequency in the pass band the dynamical tunability of the photonic band gap can be realized [4]. For frequency inside the gap, the formation of gap solitons has been demonstrated [5-9]. Furthermore, the optical bistability near the band edge $[10,11]$, the gap soliton $[5,9]$, or the defect mode [12] in finite-sized $\chi^{(3)}$ nonlinear photonic crystals has been investigated. However, how to utilize the properties of strong field localization of the gap soliton or defect mode to enhance the $\chi^{(3)}$-related nonlinear optical effects, such as the optical phase conjugation (PC), has never been explored before. As it is known, the optical PC is an important subject in nonlinear optics [13], because it has many applications such as optical signal processing, wavefront correction, etc. However, due to the very small value of $\chi^{(3)}$, the PC generation efficiency is usually very low in the conventional homogeneous media, which greatly limits its practical applications. In this paper, we demonstrate that the efficiency of PC generation can be enhanced by several orders of magnitude in a superlattice by utilizing the field localization near its band-edge, gap-soliton, or defect states. Due to the presence of feedback mechanism at each interface of a superlattice, we also demonstrate that the PC can still be effectively generated when only one pump wave is used.

Consider optical PC in a 1D nonlinear superlattice shown in Fig. 1. In this geometry, the lossless nonlinear superlattice composes of $N$ unit cells. One layer in each unit cell is linear, with the refractive index $n_{0}$ and width $l_{1}$. The other layer is nonlinear, characterized by the usual, weak-field refractive index $n$ and a third-order nonlinear susceptibility $\chi^{(3)}$. The width of the nonlinear layer is $l_{2}$. The superlattice is illuminated by two counterpropagating pump waves $\widetilde{E}_{1}$ and $\widetilde{E}_{2}$ normal to the surface of the superlattice and by a signal wave $\widetilde{E}_{3}$ incident upon the superlattice with an incident angle $\theta_{0}$. As a result of the nonlinear coupling between these waves, a new wave $\widetilde{E}_{4}$ is created that counterpropagates to the signal wave $\widetilde{E}_{3}$. In addition, because of reflections at each interface of two layers, in each layer, there appear two additional waves $\widetilde{E}_{5}$ and $\widetilde{E}_{6}$, which are the mirror reflections of $\widetilde{E}_{4}$ and $\widetilde{E}_{3}$, respectively. For simplicity, all waves are assumed to be $s$ polarized.

We represent the six interacting waves by

$$
\begin{aligned}
\tilde{E}_{j}(\vec{r}, t) & =E_{j}(\vec{r}) \exp (-i \omega t)+\text { c.c. } \\
& =A_{j}(\vec{r}) \exp \left[i\left(\vec{k}_{j} \cdot \vec{r}-\omega t\right)\right]+\text { c.c. }
\end{aligned}
$$

The total field amplitude within one nonlinear layer is given by $E=\sum_{j=1}^{6} E_{j}$. This field $E$ produces a nonlinear polarization within the layer, which is given by $P=3 \chi^{(3)} E^{2} E^{*}$. By considering only terms that have the same transverse momentum, we arrive the following nonlinear polarizations for each field:

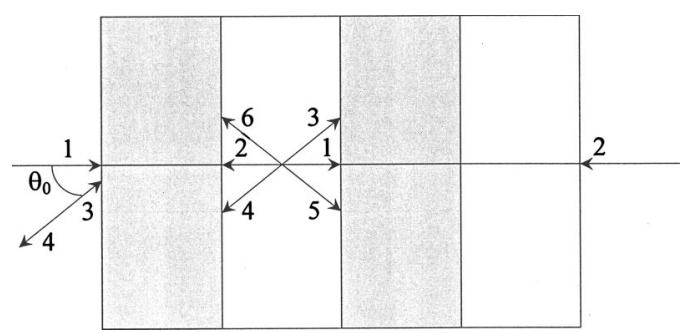

FIG. 1. Geometry for PC in a $1 \mathrm{D}$ photonic crystal with $\chi^{(3)}$ nonlinearity. $E_{1}$ and $E_{2}$ are input pump waves, $E_{3}$ is the input signal wave, and $E_{4}$ is the generated PC wave. 


$$
\begin{aligned}
P_{\alpha}= & 3 \chi^{(3)}\left\{\left(\left|E_{1}+E_{2}\right|^{2}+2 \sum_{j=3}^{6}\left|E_{j}\right|^{2}\right) E_{\alpha}\right. \\
& \left.+\left[2 E_{3} E_{4}+2 E_{5} E_{6}\right] E_{\beta}^{*}\right\}, \\
P_{\alpha^{\prime}}= & 3 \chi^{(3)}\left\{\left(2\left|E_{1}+E_{2}\right|^{2}+2 \sum_{j=3}^{6}\left|E_{j}\right|^{2}-\left|E_{\alpha^{\prime}}\right|^{2}\right) E_{\alpha^{\prime}}\right. \\
& \left.+\left[\left(E_{1}+E_{2}\right)^{2}+2 E_{5} E_{6}\right] E_{\beta^{\prime}}^{*}\right\}, \\
P_{\alpha^{\prime \prime}}= & 3 \chi^{(3)}\left\{\left(2\left|E_{1}+E_{2}\right|^{2}+2 \sum_{j=3}^{6}\left|E_{j}\right|^{2}-\left|E_{\alpha^{\prime \prime}}\right|^{2}\right) E_{\alpha^{\prime \prime}}\right. \\
& \left.+\left[\left(E_{1}+E_{2}\right)^{2}+2 E_{3} E_{4}\right] E_{\beta^{\prime \prime}}^{*}\right\},
\end{aligned}
$$

where $\alpha, \beta=1$ or $2(\alpha \neq \beta), \alpha^{\prime}, \beta^{\prime}=3$ or $4\left(\alpha^{\prime} \neq \beta^{\prime}\right)$, and $\alpha^{\prime \prime}$, $\beta^{\prime \prime}=5$ or $6\left(\alpha^{\prime \prime} \neq \beta^{\prime \prime}\right)$. By substituting Eqs. (1) and (2) into

$$
\nabla^{2} \widetilde{E}_{j}-\frac{\varepsilon}{c^{2}} \frac{\partial^{2} \widetilde{E}_{j}}{\partial t^{2}}=\frac{4 \pi}{c^{2}} \frac{\partial^{2}}{\partial t^{2}} \widetilde{P}_{j}
$$

and making the slowly varying amplitude approximation [13], we then have

$$
\frac{d A_{\alpha}}{d z}=i \kappa_{\alpha} A_{\alpha}+i \kappa_{\alpha}^{\prime} A_{\alpha+1}^{*} \quad(\alpha=1,3,5),
$$

$$
\frac{d A_{\beta}}{d z}=-i \kappa_{\beta} A_{\beta}-i \kappa_{\beta}^{\prime} A_{\beta-1}^{*} \quad(\beta=2,4,6) .
$$

Here

$$
\begin{aligned}
& \kappa_{1}=\kappa_{2}=\frac{6 \pi \omega}{n c} \chi^{(3)}\left(\left|E_{1}+E_{2}\right|^{2}+2 \sum_{j=3}^{6}\left|E_{j}\right|^{2}\right), \\
& \kappa_{\alpha}=\frac{6 \pi \omega}{n c \cos \theta} \chi^{(3)}\left(2\left|E_{1}+E_{2}\right|^{2}+2 \sum_{j=3}^{6}\left|E_{j}\right|^{2}-\left|E_{\alpha}\right|^{2}\right) \\
& (\alpha=3,4,5,6) \text {, } \\
& \kappa_{1}^{\prime}=\kappa_{2}^{\prime}=\frac{12 \pi \omega}{n c} \chi^{(3)}\left[E_{3} E_{4}+E_{5} E_{6}\right] \\
& \kappa_{3}^{\prime}=\kappa_{4}^{\prime}=\frac{6 \pi \omega}{n c \cos \theta} \chi^{(3)}\left[\left(E_{1}+E_{2}\right)^{2}+2 E_{5} E_{6}\right], \\
& \kappa_{5}^{\prime}=\kappa_{6}^{\prime}=\frac{6 \pi \omega}{n c \cos \theta} \chi^{(3)}\left[\left(E_{1}+E_{2}\right)^{2}+2 E_{3} E_{4}\right],
\end{aligned}
$$

where $\theta$ is the propagation angle of $E_{3}$ in the sample. In order to solve above equations, we divide each nonlinear layer into $M$ sublayers and assume the values of $\kappa_{j}$ and $\kappa_{j}^{\prime}$ in each sublayer to be constants [14]. In our calculation $M$ is taken so that a convergent result is reached. Solving Eq. (3) in each sublayer and using the continuity of both $E$ and $H$ (i.e., the derivative of $E$ ) at the interface of two sublayers, we finally obtain the following relations:

$$
\begin{aligned}
& {\left[\begin{array}{l}
E_{1}\left(l_{s, i}\right) \\
E_{2}\left(l_{s, i}\right) \\
E_{1}^{*}\left(l_{s, i}\right) \\
E_{2}^{*}\left(l_{s, i}\right)
\end{array}\right]^{(s, i)}=u^{(s, i)}\left[\begin{array}{l}
E_{1}\left(l_{s, i-1}\right) \\
E_{2}\left(l_{s, i-1}\right) \\
E_{1}^{*}\left(l_{s, i-1}\right) \\
E_{2}^{*}\left(l_{s, i-1}\right)
\end{array}\right]^{(s, i-1)},} \\
& u^{(s, i)}=\frac{1}{2}\left[\begin{array}{llll}
u_{11}(1+b) & u_{11}(1-b) & u_{13}(1-b) & u_{13}(1+b) \\
u_{11}^{*}(1-b) & u_{11}^{*}(1+b) & u_{23}(1+b) & u_{23}(1-b) \\
u_{13}^{*}(1-b) & u_{13}^{*}(1+b) & u_{11}^{*}(1+b) & u_{11}^{*}(1-b) \\
u_{23}^{*}(1+b) & u_{23}^{*}(1-b) & u_{11}(1-b) & u_{11}(1+b)
\end{array}\right] \text {, } \\
& {\left[\begin{array}{l}
E_{3}\left(l_{s, i}\right) \\
E_{4}\left(l_{s, i}\right) \\
E_{3}^{*}\left(l_{s, i}\right) \\
E_{4}^{*}\left(l_{s, i}\right) \\
E_{5}\left(l_{s, i}\right) \\
E_{6}\left(l_{s, i}\right) \\
E_{5}^{*}\left(l_{s, i}\right) \\
E_{6}^{*}\left(l_{s, i}\right)
\end{array}\right]=\nu^{(s, i)}\left[\begin{array}{l}
E_{3}\left(l_{s, i-1}\right) \\
E_{4}\left(l_{s, i-1}\right) \\
E_{3}^{*}\left(l_{s, i-1}\right) \\
E_{4}^{*}\left(l_{s, i-1}\right) \\
E_{5}\left(l_{s, i-1}\right) \\
E_{6}\left(l_{s, i-1}\right) \\
E_{5}^{*}\left(l_{s, i-1}\right) \\
E_{6}^{*}\left(l_{s, i-1}\right)
\end{array}\right],}
\end{aligned}
$$




$$
\nu^{(s, i)}=\frac{1}{2}\left[\begin{array}{cccccccc}
\nu_{11}(1+a) & 0 & 0 & \nu_{14}(1+a) & 0 & \nu_{11}(1-a) & \nu_{14}(1-a) & 0 \\
0 & \nu_{22}(1+a) & \nu_{23}(1+a) & 0 & \nu_{22}(1-a) & 0 & 0 & \nu_{23}(1-a) \\
0 & \nu_{32}(1+a) & \nu_{11}^{*}(1+a) & 0 & \nu_{32}(1-a) & 0 & 0 & \nu_{11}^{*}(1-a) \\
\nu_{23}^{*}(1+a) & 0 & 0 & \nu_{22}^{*}(1+a) & 0 & \nu_{23}^{*}(1-a) & \nu_{22}^{*}(1-a) & 0 \\
0 & \nu_{52}(1-a) & \nu_{53}(1-a) & 0 & \nu_{52}(1+a) & 0 & 0 & \nu_{53}(1+a) \\
\nu_{61}(1-a) & 0 & 0 & \nu_{64}(1-a) & 0 & \nu_{61}(1+a) & \nu_{64}(1+a) & 0 \\
\nu_{71}(1-a) & 0 & 0 & \nu_{52}^{*}(1-a) & 0 & \nu_{71}(1+a) & \nu_{52}^{*}(1+a) & 0 \\
0 & \nu_{64}^{*}(1-a) & \nu_{61}^{*}(1-a) & 0 & \nu_{64}^{*}(1+a) & 0 & 0 & \nu_{61}^{*}(1+a)
\end{array}\right],
$$

where $l_{s, i}$ is the width of the sublayer $i$ in the $s$ th layer,

$$
\begin{gathered}
a=\frac{n^{(s, i-1)} \cos \theta^{(s, i-1)}}{n^{(s, i)} \cos \theta^{(s, i)}}, \\
b=\frac{n^{(s, i-1)}}{n^{(s, i)}}, \quad u_{11}=\left(\cos \delta_{1}^{\prime}\right) e^{i \delta_{1}}, \\
u_{13}=i \frac{\left|\kappa_{1}^{\prime}\right|}{\kappa_{1}^{\prime *}}\left(\sin \delta_{1}^{\prime}\right) e^{i \delta_{1}}, \quad u_{23}=-i \frac{\kappa_{1}^{\prime}}{\left|\kappa_{1}^{\prime}\right|}\left(\sin \delta_{1}^{\prime}\right) e^{-i \delta_{1}}, \\
\nu_{11}=\left(\cos \delta_{3}^{\prime}\right) e^{i \delta_{3}}, \quad \nu_{14}=i \frac{\left|\kappa_{3}^{\prime}\right|}{\kappa_{3}^{\prime *}}\left(\sin \delta_{3}^{\prime}\right) e^{i \delta_{3}}, \\
\nu_{32}=-i \frac{\kappa_{3}^{\prime}}{\left|\kappa_{3}^{\prime}\right|}\left(\sin \delta_{3}^{\prime}\right) e^{-i \delta_{3}}, \quad \nu_{52}=\left(\cos \delta_{5}^{\prime}\right) e^{i \delta_{5}}, \\
\nu_{53}=i \frac{\kappa_{5}^{\prime} \mid}{\kappa_{5}^{\prime *}}\left(\sin \delta_{5}^{\prime}\right) e^{i \delta_{5},}, \quad \nu_{61}=\left(\cos \delta_{5}^{\prime}\right) e^{-i \delta_{6}}, \\
\nu_{64}=-i \frac{\kappa_{5}^{\prime}}{\left|\kappa_{5}^{\prime}\right|}\left(\sin \delta_{5}^{\prime}\right) e^{-i \delta_{6}}, \quad \nu_{71}=-i \frac{\kappa_{5}^{\prime}}{\left|\kappa_{5}^{\prime}\right|}\left(\sin \delta_{3}^{\prime}\right) e^{-i \delta_{4}},
\end{gathered}
$$

Here $\delta_{1}=l_{s, i}\left(\kappa_{1}+n^{(s, i)} k\right), \delta_{j}=l_{s, i}\left(\kappa_{j}+n^{(s, i)} k \cos \theta^{(s, i)}\right)$ for $j$ $=3,4,5,6$ and $\delta_{j}^{\prime}=\left|\kappa_{j}^{\prime}\right| l_{s, i}$ for $j=1,3,5$, where $n^{(s, i)}$ denotes the weak-field refractive index of the sublayer and $\theta^{(s, i)}$ the propagation angle of $E_{3}$ in the sublayer. For the linear layer, we take $\chi^{(3)}=0$ and $M=1$. Therefore, the electric-field amplitudes $E_{1}, E_{2}, E_{1}^{*}$, and $E_{2}^{*}$ at the output face $z=L$ and the input face $z=0$ of the superlattice are related by the transfer matrix $U=\prod_{l=0}^{N(M+1)} u^{(s, i)}$. The amplitudes $E_{3}, E_{4}, E_{3}^{*}, E_{4}^{*}$, $E_{5}, E_{6}, E_{5}^{*}$, and $E_{6}^{*}$ at $z=L$ and $z=0$ are related by the transfer matrix $V=\prod_{l=0}^{N(M+1)} \nu^{(s, i)}$, from which we obtain the output amplitude $E_{4}(0)=\left[\left(V_{28} V_{83}-V_{23} V_{88}\right) /\left(V_{22} V_{88}-V_{28} V_{82}\right)\right] E_{3}^{*}(0)$. It is clear that the wave $E_{4}$ is phase conjugate to the signal wave $E_{3}$.
Now we solve above equations numerically using an iterative procedure described in Refs. $[9,14]$. For calculations, we use a model superlattice with $N$ unit cells (i.e., $N$ nonlinear layers and $N$ linear layers), $n_{0}=1, n=2$, and $l_{1}=l_{2}=l$. For third-order nonlinear susceptibility we take $(6 \pi \omega l / n c) \chi^{(3)}$ $=-10^{-3}$. In the linear case, i.e., $\chi^{(3)}=0$, the dispersion relation gives a first band gap from $\omega=0.13386 \times 2 \pi c / l$ to $\omega$ $=0.19591 \times 2 \pi c / l$ for a normal incident light. The transmission spectrum of the light propagating normally through the model superlattice gives the transmission resonance (TR) at $\omega_{1}=0.13204 \times 2 \pi c / l$ near the lower band edge for the superlattice with $N=15$ unit cells and the TR at $\omega_{2}=0.13339$ $\times 2 \pi c / l$ for $N=30$ unit cells.

As is known, the $\chi^{(3)}$ nonlinearity has both the effect of shifting TR position or forming gap soliton [5-9] and the effect of creating PC wave $E_{4}$ [13]. Thus, in order to make the operating frequency inside the superlattice at the TR state (i.e., the band edge state or the gap soliton state), we should choose the frequency $\omega$ of the interacting waves slightly larger than $\omega_{1}$ for $N=15$ unit cells and than $\omega_{2}$ for $N=30$ unit cells. First, we consider the case of $N=15$ unit cells and choose $\omega=0.1322 \times 2 \pi c / l$. In Fig. 2 we show a typical result of output PC intensity, $\left|E_{4}(0)\right|^{2}$, as a function of input pump amplitude $\left|E_{1}(0)\right|=\left|E_{2}(L)\right|$ (curve $a$ ), where $a_{10} \equiv\left|E_{1}(0)\right|$ and $a_{40} \equiv\left|E_{4}(0)\right|$. To compare with the result in homogeneous media, in Fig. 2, we also show the PC intensity versus $a_{10}$ in a homogeneous medium of the same $\chi^{(3)}$ and length $L=30 l$ (curve $d$ ). It is interesting to see that the efficiency of the PC

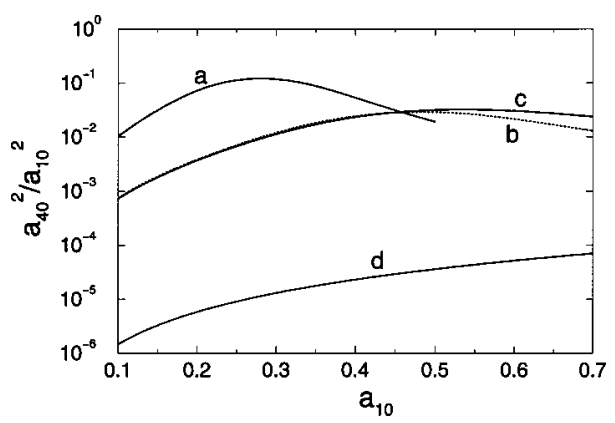

FIG. 2. $a_{40}^{2} / a_{10}^{2}$ versus $a_{10}$ in the superlattice of $N=15$ unit cells. $a_{30}=0.2$ and $\theta_{0}=2^{\circ}$. Curve $a:\left|E_{1}(0)\right|=\left|E_{2}(L)\right|=a_{10} ; \quad$ curve $b:\left|E_{1}(0)\right|=a_{10}$ and $\left|E_{2}(L)\right|=0$; curve $c:\left|E_{1}(0)\right|=0$ and $\left|E_{2}(L)\right|$ $=a_{10}$. Curve $d$ is for $a_{40}^{2} / a_{10}^{2}$ versus $a_{10}$ in a homogeneous $\chi^{(3)}$ medium of length $L=30 l$. 


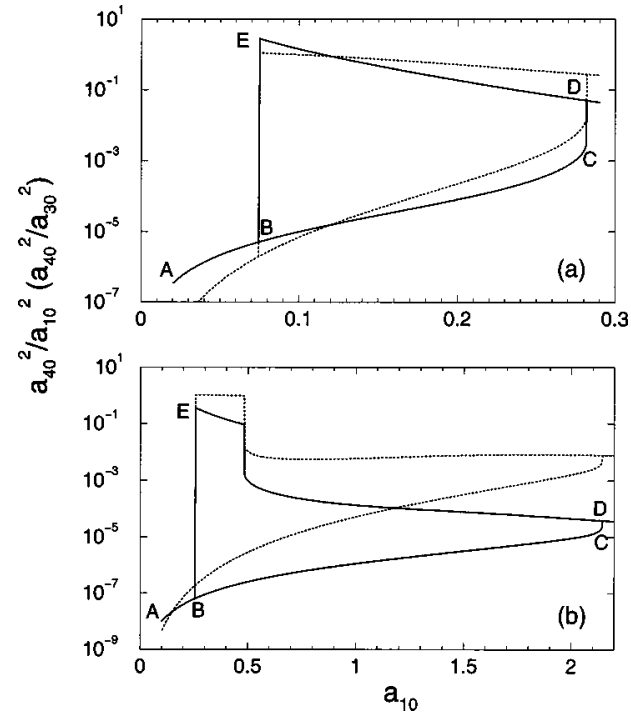

FIG. 3. $a_{40}^{2} / a_{10}^{2}$ (solid curves) and $a_{40}^{2} / a_{30}^{2}$ (dotted curves) versus $a_{10}$ in the superlattice of $N=30$ unit cells with $\theta_{0}=2^{\circ}$ and $\left|E_{1}(0)\right|$ $=\left|E_{2}(L)\right|=a_{10}$. The PC intensity follows the curve $A \rightarrow B \rightarrow C \rightarrow D$ when increasing $a_{10}$ and follows the curve $D \rightarrow E \rightarrow B \rightarrow A$ when decreasing $a_{10}$. (a) $\omega=0.1335 \times 2 \pi c / l$ and $a_{30}=0.12$. (b) $\omega$ $=0.1339 \times 2 \pi c / l$ and $a_{30}=0.15$.

generation in enhanced by more than 4 orders of magnitude compared to that in the homogeneous medium. There exists an optimal value of the input pump amplitude $a_{10}$ at which the PC generation is highest. This optimal value corresponds to the situation when the pump wave, signal wave and PC wave are approximately operating at their TR states, i.e., the shifted band-edge state. At the band-edge state, all the fields are localized inside the sample, thus providing the maximum energy exchange between the interacting waves.

Similar to the transmission behavior of a single wave through a nonlinear photonic crystal [5,9-11], as the operating frequency moves away from TR state to the band gap, the hysteresis loop of the PC intensity versus the input amplitude $a_{10}$ occurs. For example, in Fig. 3(a) we show a typical result of the output PC intensity as a function of $a_{10}$ for $\omega=0.1335 \times 2 \pi c / l$ in the model superlattice of $N=30$ unit cells. Due to the increased numbers of the nonlinear layers the efficiency of the PC generation is higher than that of $N$ $=15$ unit cells. We further verified that when the frequency moves into the band gap, the maximum efficiency of PC generation occurs via the excitation of gap soliton [5]. A typical result is shown in Fig. 3(b), where $\omega=0.1339$ $\times 2 \pi c / l$.

Because of the presence of multiple reflections in a superlattice, we expect that the $\mathrm{PC}$ wave can still be generated with only one pump beam. To verify this assertion, we take $\left|E_{1}(0)\right|=a_{10}$ and $\left|E_{2}(L)=0\right|$. Curve $b$ in Fig. 2 (dotted curve) shows a typical result when $N=15$. Curve $c$ is for $\left|E_{1}(0)\right|$

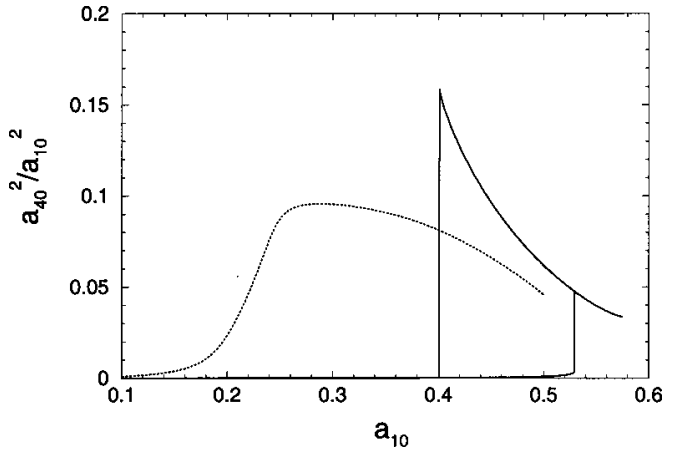

FIG. 4. $a_{40}^{2} / a_{10}^{2}$ versus $a_{10}$ in the superlattice of $N=9$ unit cells with a defect for $\omega=0.1522 \times 2 \pi c / l$ (dotted curve) and $\omega=0.1525$ $\times 2 \pi c / l$ (solid curve). $a_{30}=0.2$ and $\theta_{0}=2^{\circ}$.

$=0$ and $\left|E_{2}(L)\right|=a_{10}$, which shows the nearly same result as curve $b$. It is interesting to see that even with one-sided illumination the efficiency of PC generation is still much higher (more than 3 orders of magnitude larger) compared to that in a homogeneous medium.

We can also make use of the localized property of a defect state to enhance the efficiency of PC generation. For this purpose, we replace the middle nonlinear layer of the superlattice of $N=9$ unit cells with the nonlinear layer of width $2 l$, which produces a defect frequency at $\omega_{d}=0.1519 \times 2 \pi c / l$. We choose the operating frequency as $\omega=0.1522 \times 2 \pi c / l$. The result of $a_{40}^{2} / a_{10}^{2}$ versus $\left|E_{1}(0)\right|=\left|E_{2}(L)\right|=a_{10}$ is shown by a dotted line in Fig. 4. If the frequency $\omega$ moves further away from $\omega_{d}$ we can obtain the hysteresis loop of the PC intensity versus $a_{10}$, with a result for $\omega=0.1525 \times 2 \pi c / l$ shown by solid line in Fig. 4. This is similar to the hysteresis behavior found in transmission due to defect state [12].

The laser power required to achieve the optimal fields found in Figs. 2-4 can be quite small. For the operating frequency of $\omega=0.13 \times 2 \pi c / l$ used here, the relation $(6 \pi \omega l / n c) \chi^{(3)}=-10^{-3}$ gives $\chi^{(3)} \cong-1.3 \times 10^{-4}$ esu [15] if the units of field are chosen as statvolt $/ \mathrm{cm}$. Thus the optimal field of $a_{10} \approx 0.1-0.3$ corresponds to a laser power of $1.2-10.5 \mathrm{~W} / \mathrm{cm}^{2}$. Since the sample thickness discussed in this work is of the order of $\mu \mathrm{m}$ for optical waves, our work presents a way towards high-efficiency, low-power, microsized optical devices for signal processing.

In conclusion, we have demonstrated that by utilizing the strong field localization at the band-edge state, defect state, or single-frequency gap soliton [5] in nonlinear superlattices, the efficiency of PC generation in degenerate four-wave mixing can be enhanced by several orders of magnitude. The principle demonstrated here can actually be extended to other $\chi^{(3)}$-related nonlinear effects, such as nondegenerate two-beam coupling [16] and nondegenerate four-wave mixing [17]. In these cases, the simultaneous field localizations at different frequencies can be achieved through the formation of multifrequency gap solitons [14]. 
[1] See, for example, Photonic Band Gap Materials, edited by C. M. Soukoulis (Kluwer Academic, Dordrecht, 1996); J. D. Joannopoulos, R. D. Meade, and J. N. Winn, Photonic Crystals (Princeton University Press, Princeton, NJ, 1995); K. Sakoda, Optical Properties of Photonic Crystals (Springer-Verlag, Berlin, 2001).

[2] See, for example, C. M. Bowden and A. M. Zheltikov, J. Opt. Soc. Am. B 19, 2046 (2002), and references therein.

[3] M. Scalora et al., Phys. Rev. A 56, 3166 (1997); Opt. Photonics News 38 (2001); A. V. Andreev et al., J. Opt. Soc. Am. B 19, 2083 (2002); A. M. Malvezzi et al., ibid. 19, 2122 (2002).

[4] M. Scalora et al., Phys. Rev. Lett. 73, 1368 (1994); P. Tran, Opt. Lett. 21, 1138 (1996).

[5] W. Chen and D. L. Mills, Phys. Rev. Lett. 58, 160 (1987).

[6] C. M. de Sterke and J. E. Sipe, in Progress in Optics XXXIII, edited by E. Wolf (Elsevier, Amsterdam, 1994), Chap. III.

[7] S. John and N. Akozbek, Phys. Rev. Lett. 71, 1168 (1993); Phys. Rev. E 57, 2287 (1998).
[8] S. F. Mingaleev and Y. S. Kivshar, Phys. Rev. Lett. 86, 5474 (2001).

[9] P. Xie, Z. Q. Zhang, and X. Zhang, Phys. Rev. E 67, 026607 (2003); P. Xie and Z. Q. Zhang, ibid. 69, 036601 (2004).

[10] B. Xu and N. B. Ming, Phys. Rev. Lett. 71, 1003 (1993); B. $\mathrm{Xu}$ and N. B. Ming, ibid. 71, 3959 (1993).

[11] Z. L. Wang et al., Phys. Rev. B 53, 6984 (1996).

[12] E. Centeno and D. Felbacq, Phys. Rev. B 62, R7683 (2000).

[13] See, for example, R. W. Boyd, Nonlinear Optics (Academic Press, New York, 1992), Chap. 6.1, pp. 241-257.

[14] P. Xie and Z. Q. Zhang, Phys. Rev. Lett. 91, 213904 (2004).

[15] The magnitude of $\chi^{(3)}$ so chosen is smaller than that of GaAs $\left(6.5 \times 10^{-4}\right.$ esu). See p. 163 of Ref. [13].

[16] See, for example, Nonlinear Optics (Ref. [13]) Chap. 6.4, pp. 269-274.

[17] Y. R. Shen, The Principles of Nonlinear Optics (Wiley, New York, 1984), Chap. 14.2, pp. 247, 248. 